\section{rev Psi}

Revista de Psicología (UNLP)

https://revistas.unlp.edu.ar/revpsi

\title{
Modificación de memorias maladaptativas durante el sueño y la vigilia: una visión interdisciplinaria
}

\author{
Matías Bonilla ${ }^{1}$ Camila Isabel Jorge ${ }^{1}$ Malen Daiana Moyano ${ }^{1}$ Cecilia Forcato ${ }^{1}$ \\ Correspondencia \\ cforcato@itba.edu.ar
}

Filiaciones institucionales

${ }^{1}$ Instituto Tecnológico de Buenos Aires (ITBA)

(Argentina)

\section{Resumen}

Las memorias consolidadas pueden atravesar por un período de labilidad frente a la presentación de recordatorios (claves ligadas al aprendizaje inicial), seguido de un proceso de re-estabilización conocido como reconsolidación. Por otro lado, el sueño tiene un rol activo en la formación y modificación de memorias, así como en la reducción del tono emocional de las experiencias. Durante el mismo, las nuevas memorias se reactivan, refuerzan e integran a las redes mnésicas preexistentes. Dentro del contexto terapéutico, se pueden evocar memorias antiguas según la necesidad del sujeto, lo que podría estar desencadenando constantes labilizaciones/reestabilizaciones, quizás sin ser conscientes de ello. En la presente revisión bibliográfica discutimos los avances neurocientíficos relacionados a la reactivación y modificación de memorias durante la vigilia y el sueño, así como los últimos desarrollos en terapias psicoterapéuticas para trastornos de ansiedad, con el objetivo de pensar una práctica más interdisciplinaria.

\section{Palabras clave}

reactivación | memoria emotiva | sueño | trastornos de ansiedad

\section{Cómo citar}

Bonilla, M., Jorge, C. I., Moyano, M. D.

y Forcato, C. (2021). Modificación de memorias maladaptativas durante el sueño y la vigilia: una visión interdisciplinaria. Revista de Psicología, 20(1), 221-242. doi: 10.24215/2422572XE073

$\begin{array}{lll}\text { Recibido } & \text { Aceptado } & \text { ISSN } \\ 9 \text { jun. } 2020 & 12 \text { nov. } 2020 & 2422-572 X \\ \text { Publicado } & & \text { Licencia } \\ 20 \text { nov. } 2020 & & \text { Licencia de Cultura Libre CC-BY 4.0 } \\ & & \text { (Compartir - Adaptar - Atribuir) }\end{array}$

\section{Entidad editora}

RevPsi es una publicación de la Facultad de Psicología (Universidad Nacional de La Plata, Argentina) 


\section{Análise dos efeitos da cronificação nos usuários admitidos em uma instituição psiquiátrica monovalente}

\section{Resumo}

As memórias consolidadas podem passar por um período de labilidade diante da apresentação de lembretes (chaves vinculadas ao aprendizado inicial), seguidos por um processo de reestabilização conhecido como reconsolidação. Por outro lado, o sono tem um papel ativo na formação e modificação de memórias, bem como na redução do tom emocional das experiências. Durante isso, as novas memórias são reativadas, reforçadas e integradas às redes de memória pré-existentes. No contexto terapêutico, velhas lembranças podem ser evocadas de acordo com a necessidade do sujeito, o que pode estar desencadeando constantes desestabilizações reestabilizações, talvez sem ter consciência disso. Nesta revisão bibliográfica, discutimos os avanços neurocientíficos relacionados à reativação e modificação de memórias durante a vigília e o sono, bem como os últimos desenvolvimentos em terapias psicoterapêuticas para transtornos de ansiedade, com o objetivo de pensar em uma prática mais interdisciplinar.

\section{Palavras-chave}

reativação | memória emocional | sono | transtornos de ansiedade

\section{Modification of maladaptive memories during sleep and wakeful- ness: an interdisciplinary perspective}

\section{Abstract}

Consolidated memories can return to a labile state after a reminder presentation (cue associated to the initial learning) followed by a re-stabilization process known as reconsolidation. Furthermore, sleep has an active role in the formation and modification of memories, as well as in decreasing the emotional tone of the experiences. During sleep, new memories are reactivated, reinforced and integrated into pre-existing networks. Within the therapeutic context, old memories can be retrieved according to the subject's needs, and this could be triggering repeated labilization/restabilization processes, perhaps without being aware of it. In this review we discuss the neuroscientific advances regarding memory reactivation and modification during sleep and wakefulness, as well as the latest approaches in psychotherapeutic therapies for anxiety disorders, with the aim of thinking about a more interdisciplinary practice.

\section{Keywords}

reactivation | emotional memory | sleep | anxiety disorders 


\section{Aspectos destacados del trabajo}

- Los procesos de consolidación y reconsolidación de la memoria son procesos imprescindibles a tener en cuenta a la hora de diseñar intervenciones terapéuticas.

- El estudio del sueño en trastornos de ansiedad representa un aspecto crucial a la hora de pensar los cuadros clínicos.

- La inclusión del sueño como herramienta terapéutica en trastornos psiquiátricos/ psicológicos conlleva mejoras significativas y estables.

- En el diseño de nuevas estratégicas terapéuticas es fundamental tener en cuenta el tipo de memoria en cuestión, las ventanas temporales entre la reactivación de la memoria y la intervención, así como el tiempo entre la intervención y el momento de dormir.

La memoria es una de las funciones cognitivas más importantes que tienen los seres vivos, otorgándoles la capacidad de adaptarse a los cambios del ambiente. El sueño tiene un rol fundamental tanto en la formación como en la modificación de la información almacenada, permitiendo no sólo el reprocesamiento e integración de las nuevas memorias sino, al mismo tiempo, la reducción del tono emocional ligado a las experiencias, y, por tanto, la posterior evocación de un episodio sin la carga emotiva de lo vivido (Rasch y Born, 2013; Walker y Van der Helm, 2009). Sin embargo, en ciertas oportunidades, puede darse lugar a la formación de memorias maladaptativas que perjudican la adaptación al medio y, por consiguiente, la calidad de vida de un individuo.

\section{El sueño}

El sueño se define como un estado natural y reversible, durante el cual la respuesta a los estímulos externos se encuentra disminuida. Representa un período de inactividad, acompañado de pérdida de conciencia. El sueño ocurre a intervalos regulares y está regulado homeostáticamente; es decir, una pérdida o retraso del sueño resulta en un sueño prolongado posterior (Borbély et al., 2016). Se ha demostrado que la gran mayoría de los organismos duermen, desde moluscos, insectos y peces, hasta aves y mamíferos (Vorster y Born, 2015).

Los humanos, cuando dormimos, lo hacemos en ciclos aproximados de 90 minutos. En una noche, podemos atravesar entre cuatro a seis ciclos y cada uno de ellos está formado por sueño de movimientos oculares rápidos (MOR) y sueño no-MOR. El sueño no-MOR se divide a su vez en sueño ligero (estadios 1 y 2) y sueño profundo 
o sueño de ondas lentas (estadios 3 y 4) (Rechtschaffen y Kales, 1968). Durante este período el cerebro muestra un metabolismo global reducido (Braun et al., 1997), una actividad eléctrica cortical sincronizada y bi-estabilidad generalizada de los potenciales de membrana corticales (Steriade et al., 2001). Es decir, la corteza oscila entre un período de mínima actividad (hiperpolarización de la corteza) y uno de máxima actividad (despolarización de la corteza). Así, el sueño profundo se caracteriza por la presencia de ondas lentas corticales $(0.5-1 \mathrm{~Hz})$, de husos de sueño hipocampo-corticales $(8-15 \mathrm{~Hz})$ y complejo de ondas agudas hipocampales (Sharp Wave-ripples, 80-100 Hz en humanos) (Diekelmann y Born, 2010). A su vez, la actividad neuromodulatoria muestra una caída en la actividad colinérgica, noradrenérgica y de cortisol (Born y Fehm, 1998; McCarley, 2007). Por el contrario, durante el sueño MOR la corteza muestra una actividad global desincronizada, similar a la vigilia, principalmente producida por las proyecciones colinérgicas del tronco cerebral, acompañada por niveles similares de flujo sanguíneo y actividad metabólica (Buchsbaum et al., 1989; Velázquez-Moctezuma et al., 1991). Durante el MOR, los músculos esqueléticos del cuerpo son suprimidos activamente por las estructuras neuronales en el tronco cerebral, con excepción de los que controlan el diafragma, el movimiento ocular y el oído medio (Hobson et al., 2000). Está caracterizado por ondas pontogeniculadas occipitales (PGO) y actividad oscilatoria predominante theta (Diekelmann y Born, 2010). La neurotransmisión noradrenérgica es nula mientras que la actividad colinérgica se incrementa, al igual que la liberación de cortisol (Born y Fehm, 1998, McCarley, 2007).

Se han asociado diversos roles funcionales al sueño, entre los que se encuentran: restaurador de las fuentes de energía (Berger y Phillips, 1995; Webb, 1988), reparación de tejidos (Oswald, 1980), termorregulación (Rechtschaffen y Bergmann, 1995), regulación metabólica (Knutson et al., 2007; Van Cauter et al., 2008), limpieza del cerebro de radicales libres y proteínas aberrantes -como la proteína beta-amiloide (Varga et al., 2016)-, función inmunológica (Lange et al., 2010) y a su vez el sueño también se encuentra involucrado en la plasticidad sináptica y los procesos de memoria (Rasch y Born, 2013; Tononi y Cirelli, 2006).

\section{La memoria}

Según su duración en el tiempo, las memorias pueden clasificarse en: memorias de corto plazo, que duran desde segundos a minutos y memorias de largo plazo que pueden durar horas, meses e incluso toda la vida (Kandel, 2000). Las memorias de largo plazo, a su vez, pueden clasificarse en declarativas y no declarativas (Squire, 2015). Las primeras son representaciones internas de hechos y episodios que son accesibles a la recolección consciente (Dudai, 2004) y dependen principalmente de la participación del hipocampo y estructuras aledañas al lóbulo temporal medio al momento de ser evocadas (Squire y Zola, 1996). Las no declarativas en cambio, no requieren de una recolección consciente y son independientes del hipocampo (Dudai, 2004). Sin embargo, en los últimos años esta clasificación está siendo puesta 
en duda ya que memorias con contenido declarativo pueden ser adquiridas de forma subliminal (Duss et al., 2014; Henke, 2010; Reber et al., 2014; Reber y Henke, 2011; Wuethrich et al., 2018). Más aún, recientemente se ha observado la participación del hipocampo en la reactivación de memorias no declarativas (Jacobacci et al., 2020). A su vez, las memorias declarativas se dividen en memorias semánticas y episódicas. La memoria semántica se refiere al conocimiento de conceptos, de sus relaciones, de las reglas para su uso, así como al conocimiento general acerca del mundo cuando tal conocimiento no está ligado al contexto espacio-temporal de su adquisición. Por otro lado, la memoria episódica contiene información de acontecimientos contextuados, acotados espacial y temporalmente (Tulving, 1972).

La memoria puede ser dividida en diferentes subprocesos entre los que se encuentran: la adquisición, la consolidación, la evocación, la reconsolidación, la extinción y el olvido. La adquisición representa el nacimiento de la memoria y refiere a la codificación de información en circuitos neuronales. Luego de la adquisición, la memoria atraviesa un período de labilidad, en la cual es susceptible a la acción de agentes amnésicos o facilitadores, seguido por un proceso de estabilización conocido como consolidación. Este subproceso consiste en el pasaje de la información desde un estado lábil a uno estable; representa el almacenamiento de la información, la cual depende de la síntesis proteica y de la expresión génica (Dudai y Morris, 2000). La evocación implica la recuperación de la información previamente almacenada. Hasta no hace mucho tiempo se consideraba que una vez consolidada, la memoria no podía ser modificada. Sin embargo, se observó que la misma puede volver a un estado de labilidad luego de la exposición a claves asociadas al aprendizaje inicial. Esta fase se encuentra seguida de un proceso de reestabilización conocido como reconsolidación, dependiente de síntesis proteica y expresión génica (Nader et al., 2000). Así, frente a la presentación de un recordatorio pueden ocurrir dos procesos: evocación sin labilización, dónde solo se recupera la memoria almacenada, o labilización/reestabilización de la memoria, que permite modificar la traza de memoria (Forcato et al., 2009; Pedreira et al., 2004; Sevenster et al., 2013). Por ejemplo, los recordatorios incompletos formados por una parte de la asociación aprendida (ej: sílaba- clave sin sílaba-respuesta en el protocolo de sílabas asociadas) son más eficientes que los recordatorios completos (ej: sílabaclave más la sílaba-respuesta presente) para desencadenar el proceso de labilizaciónreconsolidación (Forcato, 2009). Este hecho se adjudica a que para desencadenar la labilización-reconsolidación de una memoria es necesario que se produzca un error en la predicción, es decir, una incongruencia entre lo aprendido y lo que ocurre durante la reactivación (Sinclair y Barense, 2019). El proceso de reconsolidación representa una oportunidad única para modificar la información almacenada y no sólo se demostró que, una vez labilizada, la memoria original puede ser perjudicada, sino que, a su vez, la misma puede ser mejorada o puede incorporarse dentro de ella nueva información (Forcato et al., 2007; Hupbach et al., 2007; Nader et al., 2013). Por otro lado, la presentación repetida de un recordatorio así como la re-exposición prolongada a un contexto, puede desencadenar el proceso de extinción. La misma implica la caída 
en la respuesta adquirida y da lugar a la formación de una nueva memoria que inhibe a la memoria original, ganando esta última el control del comportamiento (Myers y Davis, 2002). Dado que la extinción implica la formación de una nueva memoria que compite con la original, bajo ciertas circunstancias la memoria original puede volver a tomar el control del comportamiento. Esto puede darse con 1) el simple pasaje del tiempo (recuperación espontánea); 2) el cambio de contexto (renovación) o 3) la presentación del estímulo incondicionado (reinstalación) (Bouton, 2002; Myers y Davis, 2002). Por último, el olvido fue inicialmente entendido como una caída en la respuesta adquirida, debido al pasaje del tiempo y falta de evocación de la información de interés (Ebbinghaus, 1885). Hoy en día se sabe que el olvido es un proceso activo y que en gran medida puede ser explicado por la interacción entre las memorias al momento de la evocación. Dado que las memorias no son almacenadas de manera independiente unas de otras, sino en complejas redes de asociación, la evocación de un trazo puede inhibir temporalmente la evocación de una memoria relacionada; un proceso conocido como olvido inducido por evocación (Anderson et al., 1994). Si esta inhibición se repitiese en el tiempo, podría explicar el olvido permanente de cierta información. Por otro lado, se ha demostrado que deliberadamente se puede inhibir la evocación de memorias no deseadas o incluso disminuir la adquisición de cierta información; un proceso denominado olvido motivado, en el cual interviene el control inhibitorio prefrontal y podría ser otro motivo de por qué olvidamos información (Anderson y Hanslmayr, 2014).

\section{Sueño y memoria}

\section{Consolidación activa de la memoria durante el sueño}

Desde los inicios del estudio sobre el sueño y la memoria, se observa que el sueño posterior a la adquisición de nueva información mejora la consolidación de la misma. Inicialmente, se planteó que el sueño cumplía un rol protector contra las interferencias del medio, dado que al dormir la codificación de información se encuentra reducida (Ebbinghaus, 1885). Sin embargo, hoy en día se postula que el sueño tiene un rol activo en la formación y modificación de memorias (Rasch y Born, 2013).

La hipótesis de la consolidación activa durante el sueño, basándose en la "Modelo Estándar de la Consolidación en dos Etapas" (Marr, 1971; Buzsáki, 1989), asume la existencia de dos centros de almacenamiento: el hipocampo, que permite aprender a un ritmo rápido y sirve como un búfer intermedio que almacena la información temporalmente; y la neocorteza que permite aprender más lentamente, pero almacena la información a largo plazo (Diekelmann y Born, 2010). Inicialmente, los nuevos eventos se codifican en paralelo en ambos centros y, durante el sueño de ondas lentas, la información recientemente adquirida se reactiva espontáneamente en el hipocampo, transfiriéndose hacia la neocorteza y redistribuyéndose en circuitos cortico-corticales, favoreciendo así su consolidación a largo plazo. Sin bien inicialmente esta hipótesis fue planteada para la memoria declarativa, puede 
aplicarse a cualquier sistema de memoria (Diekelmann y Born, 2010). A su vez, la hipótesis plantea que las ondas lentas durante el sueño de ondas lentas (SOL) orquestan el diálogo hipocampo-cortical, impulsando la reactivación de la memoria en el hipocampo a través de los complejos de ondas agudas hipocampales (Sharp Wave Ripples), junto con los husos de sueño tálamo-corticales, que participan en la inducción cambios plásticos duraderos en las áreas corticales (Rasch y Born, 2013). Así, durante el SOL se reactivaría la información previamente adquirida y durante el subsecuente MOR se estabilizaría e integraría con las redes de memoria preexistentes (Born y Wilhelm, 2012; Diekelmann y Born, 2010; Rasch y Born, 2013).

Por otro lado, la hipótesis de la homeostasis sináptica, plantea que el sueño es el precio que el cerebro "paga" por la plasticidad. La codificación constante de información que ocurre durante la vigilia aumenta la fuerza sináptica en el cerebro. Esto produce un incremento en los requerimientos energéticos celulares y disminuye la relación señal-ruido, saturando así al aprendizaje. Durante el sueño, principalmente durante el de ondas lentas, se produce una restauración de las sinapsis restableciendo la homeostasis celular (Tononi y Cirelli, 2014). Así, el sueño tendría un rol fundamental en la "limpieza" de las sinapsis, lo que explicaría por qué al despertar estaríamos preparados para una nueva ronda de aprendizaje (Diekelmann y Born, 2010).

\section{Dormir para olvidar, dormir para recordar}

Una de las razones que explica por qué las memorias emocionales parecen estar codificadas y consolidadas de forma más robusta que las memorias neutras, se debe a los neurotransmisores implicados en el momento de la experiencia ( $M c G a u g h$, 2004). Sin embargo, se observa que la evocación posterior de la memoria carece de la magnitud autonómica generada durante la adquisición. Walker y Van der Helm (2009) postularon que el sueño MOR presentaría las condiciones neuroanatómicas, neurofisiológicas y neuroquímicas óptimas para el procesamiento de este tipo de memorias. La hipótesis de "dormir para olvidar, dormir para recordar" (Walker y Van der Helm 2009) plantea que durante la vigilia la formación de una memoria episódica involucra la codificación coordinada hipocampo-cortical facilitada por la amígdala y modulada por altas concentraciones de noradrenalina. Durante el sueño MOR posterior, estas mismas estructuras neuronales se reactivan, coordinadas por la actividad oscilatoria theta, permitiendo el reprocesamiento de experiencias emocionales previamente aprendidas. Sin embargo, esta reactivación ocurre en un medio neuroquímico desprovisto de modulación noradrenérgica; recordemos que durante el sueño MOR la actividad noradrenérgica es nula. Como consecuencia, el reprocesamiento de la memoria emocional puede lograr, por un lado, una disminución del tono emocional inicialmente asociado con el evento durante la codificación; mientras que, por otro, una consolidación neocortical simultánea y progresiva de la información. Así, el sueño MOR facilitaría la consolidación e integración de memorias de eventos emocionales y, al mismo tiempo, ayudaría a disminuir el tono emocional de un recuerdo (Walker y Van del Helm, 2009; van del Helm y Walker, 2010). 


\section{Trastornos de ansiedad}

En este apartado introducimos los Trastornos de Ansiedad (TA) dado que, desde la neurociencia, podrían considerarse como memorias emocionales maladaptativas. Los dos elementos principales que los constituyen son el miedo y la ansiedad. El primero, es una emoción primaria crítica para la supervivencia (Ekman y Friesen, 2003). El mismo desencadena un estado neurofisiológico automático primitivo de alarma que prepara al individuo para escapar o defenderse del supuesto estímulo amenazante y su funcionamiento se relaciona con procesos automáticos que ocurren en la amígdala. Por ejemplo, si mientras caminamos por una calle oscura de noche se vislumbra la silueta de una persona a lo lejos, nuestro cuerpo comienza a sufrir distintos procesos automáticos que nos preparan para el escape, en caso de tratarse de una amenaza real. Por otro lado, la ansiedad, que es una respuesta anticipatoria que aparece frente a una amenaza futura que puede ser real o imaginada (American Psychological Association, 2013). Se activa al anticipar circunstancias o sucesos que se interpretan como aversivos, incontrolables y que podrían, potencialmente, amenazar los intereses vitales del individuo.

Los TA se caracterizan por respuestas excesivas y prolongadas de ansiedad y miedo frente a distintos estímulos. Comparten características de miedoy ansiedad excesivos, así como también alteraciones conductuales asociadas a estos. Se diferencian de los mecanismos normales de ansiedad por su excesiva duración (+/- 6 meses). Muchos de ellos se desarrollan en la infancia y suelen persistir si no son tratados (American Psychological Association, 2013).

A partir de lo mencionado, se desprende que el miedo es la base de cualquier trastorno de ansiedad -ya que es la valoración automática de base del peligro-, mientras que la ansiedad es un estado más duradero de la amenaza e incluye otros factores cognitivos como el pensamiento, el lenguaje, la percepción, la memoria, el razonamiento, la atención, la resolución de problemas o la toma de decisiones (Clark y Beck, 2012)

La sintomatología de los TA se puede resumir en tres grupos: conductual (producen cambios en nuestra forma de actuar), cognitivo (cambios en la forma de pensar o de percibir el contexto) y fisiológico (distintas respuestas fisiológicas como palpitaciones, sequedad en la boca, mareos, entre otros) (Clark y Beck, 2012). Por lo tanto: ¿a partir de cuándo el miedo y la ansiedad se tornan factores maladaptativos que conllevan a la aparición de un trastorno que requiere de intervención clínica?

Clark y Beck (2012) sugieren cinco criterios para distinguir los estados anormales de miedo y de ansiedad. No es necesario que todos ellos se encuentren presentes, pero sí se espera que muchas de estas características lo estén en un cuadro clínico.

1. Cognición disfuncional: este criterio se desprende de la teoría cognitiva (Beck, 1985) y refiere a la activación de esquemas mentales inadaptativos (creencias) y a los errores en el proceso cognitivo que, asociados a un miedo excesivo, no condicen con la realidad objetiva de la situación. 
2. Deterioro del funcionamiento: la ansiedad clínica interfiere directamente con la vida diaria del individuo, repercutiendo en su vida social y en su desempeñolaboral.Distintassituaciones pueden desencadenar respuestas de miedo a partir de patrones adquiridos en las situaciones traumáticas. Muchas veces, las respuestas de miedo pueden ser contraproducentes ya que las mismas aumentan el riesgo del daño o peligro. Por ejemplo, una persona que sufrió un accidente de tráfico tendría un estado de ansiedad mucho más elevado al volver a manejar un auto, provocando que no esté muy atenta al tráfico, ya que estaría más preocupada por revisar constantemente el espejo retrovisor, aumentando así las posibilidades de provocar un accidente de tránsito.

3. Persistencia: cuando se habla de un estado clínico, la ansiedad persiste mucho más de lo que podría esperarse en condiciones normales. Las personas son propensas a sufrir de ansiedad, experimentándola a diario, durante años.

4. Falsas alarmas: miedo o pánico visible sin un estímulo amenazante, aprendido o no aprendido. La crisis de angustia espontánea, sin estímulo que la genere, es el mejor ejemplo de este tipo de alarmas.

5. Hipersensibilidad a los estímulos: el miedo es una respuesta provocada por un estímulo, externo o interno, que se percibe como amenaza potencial. En un cuadro clínico, el miedo es provocado por una amplia gama de estímulos o situaciones en las que la intensidad de la amenaza es relativamente leve y podría hasta clasificarse como inocua.

Habiendo definido las características de los trastornos de ansiedad, resulta pertinente aclarar que existen varios subtipos y que, si bien todos ellos comparten algunas características fundamentales -como la activación del miedo-, las diferencias externas son muy importantes ya que para cada tipo de trastorno de ansiedad existe un tratamiento específico. Algunos de estos trastornos son: Trastorno Obsesivo Compulsivo (TOC), Trastorno por Estrés Post Traumático (TEPT), Trastorno de Pánico (TP), Trastorno de Ansiedad Generalizada (TAG), Fobia Social, Agorafobia y Trastorno de Fobia específica. A continuación, definiremos dos de los trastornos más estudiados desde una perspectiva neurocientífica:

- Trastorno de fobia específica: se define como la aparición de miedo o ansiedad intenso y persistente, prácticamente inmediata e invariable respecto de una situación o de un objeto específico que se evita o se soporta a costa de un miedo o una ansiedad intensa. Por ejemplo, alguien que sufre de agorafobia puede evitar participar de eventos concurridos o en caso de que asista tendrá niveles de ansiedad elevados. Los síntomas deben prevalecer, por lo menos, por seis meses (American Psychological Association, 2013). 
- Trastorno de estrés post-traumático (TEPT): este es un fenómeno que se presenta a partir de la exposición, real o imaginada, a un evento, que haya resultado traumático en la propia persona o en terceros (American Psychological Association, 2013). Se caracteriza por generar en la persona síntomas de ansiedad extremos (síntomas de reviviscencia, de evitación, de hipervigilancia, ataques de pánico, etc). Estos pueden desaparecer con el tiempo o persistir, requiriendo de asistencia médica. Además, también presentan alteraciones cognitivas negativas, alteraciones del estado de ánimo, síntomas de intrusión, así como también alteración de la reactividad relacionados al evento traumático. También, mecanismos de evitación persistente de estímulos asociados al evento. En el marco de lo estudiado, esto último nos resulta relevante, ya que nos indica que el trastorno de estrés postraumático es susceptible a la presentación de claves. Por último, se pueden mencionar trastornos del sueño en la mayoría de los pacientes, que incluyen pesadillas, dificultad para conciliar el sueño, así como también sueño interrumpido (American Psychological Association, 2013).

\section{Inducción de reactivaciones de la memoria durante la vigilia}

Dependiendo del tratamiento otorgado luego de la presentación de un recordatorio durante la vigilia, se puede desencadenar tanto una caída en la memoria original como una mejora o una incorporación de nueva información dentro del mismo trazo de memoria (Forcato et al., 2007, 2010, 2011, 2013). Así, la presentación de segundos aprendizajes que compartan los mismos sistemas de memoria que la memoria original, interfieren en la reestabilización de la memoria, observándose, en estos casos, una caída en el desempeño durante la evaluación (Fernández et al., 2016a). Por otro lado, hemos evidenciado que la presentación consecutiva de al menos dos recordatorios que labilizan la memoria declarativa desencadena el reforzamiento de la misma 24hs. después, mejorando la precisión de la memoria (Forcato et al., 2011), mientras que la presentación de al menos un recordatorio es suficiente para aumentar la persistencia de la memoria una semana después (Forcato et al., 2013, 2014). A su vez, si una nueva información es presentada luego de la reactivación, la misma puede ser incorporada como parte de la memoria original (Forcato et al., 2010). Así, dadas estas características únicas del proceso de reconsolidación, que permite la modificación de las memorias almacenadas, se plantea al mismo como una posible herramienta para el tratamiento de memorias maladaptativas, como lo son los trastornos de ansiedad (Alberini y Ledoux, 2013; Lane et al., 2015).

Una de las terapias más comúnmente utilizada en la clínica es la terapia de exposición (Foa y Kozak, 1986). La misma es de gran utilidad clínica, proviene de la teoría cognitivo-conductual y es la primera opción indicada según guías de tratamientos eficaces, tales como la creada por la división 12 proporcionada por la American Psychological Association (American Psychological Association, 2020). Su objetivo es el tratamiento de trastornos y condiciones basadas en respuestas maladaptativas 
sobre las que el sujeto pierde el control y generan tal grado de malestar que imposibilitan el accionar diario. Básicamente se trata de exponer al sujeto de manera deliberada a las situaciones promotoras de ansiedad o malestar para que él mismo pueda experimentarlas sin la realización de la conducta que busca la disminución del nivel de ansiedad (conducta de evitación). Desde las neurociencias, la terapia de exposición puede ser interpretada como un aprendizaje de extinción (Schiller et al., 2010). Es decir, esta terapia formaría una nueva memoria que inhibe a la memoria original, ganando temporalmente el control del comportamiento. Este hecho explicaría, por ejemplo, por qué algunos pacientes presentan recaídas con el pasaje del tiempo (recuperación espontánea), aunque cabe destacar, sin embargo, que la tasa de recaídas es baja (Bouton, 2002). Esto puede deberse al hecho de que los individuos suelen mantener un contacto con su terapeuta en aquello que se denomina sesiones de mantenimiento, las cuales podrían estar conservando activa la memoria de extinción; es decir, favoreciendo la inhibición de la memoria original.

En contraposición a la extinción, la utilización de la reconsolidación como herramienta para la modificación de memorias maladaptativas propone la apertura y modificación permanente del trazo mnésico, sin la posibilidad de recaídas dado que la memoria original se encuentra alterada. En esta línea, Schiller et al. (2010) utilizaron a la reconsolidación en combinación con la extinción con el objetivo de extinguir una memoria aversiva previamente labilizada. Los participantes fueron entrenados, el primer día, con tres estímulos visuales (cuadrados de colores), uno de los cuales estaba asociado a la presentación de un choque eléctrico. Al segundo día, se presentó el recordatorio, el cual estaba conformado por un cuadrado de color que el día anterior se asoció a un choque eléctrico, pero en este caso se presentó en ausencia del mismo. A los 10 min o 6 hs. después de haber sido presentado el recordatorio se realizó un protocolo de extinción. A su vez, se corrió un grupo control, el cual no recibió recordatorio, pero sí el entrenamiento de extinción. Al tercer día, se les aplicó nuevamente un protocolo de extinción en donde mediante la medición del nivel de electroconductancia de la piel, se evaluó la recuperación espontánea del miedo mientras se les presentaba el estímulo que estuvo previamente asociado al choque eléctrico, pero, esta vez, en ausencia de él. Lo que observaron fue que solo el grupo que había recibido el protocolo de extinción 10 min. después de la reactivación no mostró recuperación espontánea del miedo durante la evaluación. Es decir, a los $10 \mathrm{~min}$. la memoria original aún se encontraba lábil, pudiéndose modificar el contenido y resignificando así el estímulo previamente asociado al choque, dentro de la misma red de memoria. Por otro lado, a 6 horas de la reactivación la memoria ya se encontraba reconsolidada; por lo tanto, el protocolo de extinción formó una segunda memoria que competía por el control del comportamiento, observándose así recuperación espontánea de la memoria original en el tercer día. Esta modificación de la red original es dependiente de reactivación, dado que en el grupo que no recibió recordatorio también se observó recuperación espontánea. Este trabajo deja en evidencia que la terapia de exposición podría combinarse con la reactivación de la memoria original a fin de ser utilizada en la clínica, permitiendo una labilización y modificación permanente del trazo mnésico original. 
Ahora bien, es importante analizar estos descubrimientos a la luz de memorias episódicas, tales como las que se manejan en un contexto psicoterapéutico. Schwabe y Wolf (2009) crearon un paradigma en donde se les pedía a los voluntarios que recordasen una memoria autobiográfica de no más de dos semanas de antigüedad. Debían recordar mediante adjetivos que se les presentaban y funcionaban como disparadores de tres tipos de memorias: memorias aversivas, memorias positivas y memorias neutras. Inmediatamente después de recordarlas, debían aprender el pasaje de un libro. Una semana después fueron evaluados. Observaron que aquellos que habían aprendido la historia (tarea interferente) después de haber recordado la memoria autobiográfica neutra recordaban menos detalles que para las memorias emotivas. Es decir, la tarea interferente solo perjudicó la reestabilización de las memorias neutras y este efecto fue dependiente de la presentación del recordatorio, dado que no se evidenció al aprender la tarea interferente sin la presentación del recordatorio (Schwabe y Wolf, 2009). En 2012, el mismo grupo investigó la posibilidad de interferir la reconsolidación de memorias emotivas con Propranolol, un antagonista de los receptores adrenérgicos beta 1 y 2 . Observaron entonces que aquellos sujetos a los que se les había suministrado el Propranolol redujeron significativamente su respuesta de miedo frente al estímulo aversivo en comparación con el grupo placebo (Schwabe et al., 2012). Estos hallazgos apoyan a los reportados previamente por Brunet et al. (2008), en donde se administró Propranolol luego de la reactivación de memorias aversivas en pacientes con TEPT. Se observó que los síntomas fisiológicos suscitados por la memoria aversiva fueron significativamente disminuidos, demostrando así los efectos del Propranolol como agente interferente de la reconsolidación de la memoria (Brunet et al., 2008).

Cabe destacar que resulta fundamental realizar estudios que involucren seguimiento a largo plazo para evaluar su aplicación en la clínica. En 2015, Soeter y Kindt (2015) estudiaron la reconsolidación en pacientes con fobia a las arañas. Lo interesante de este estudio es que la memoria misma de la fobia era reactivada mediante la exposición a una tarántula que se encontraba en un terrario. Inmediatamente después de la reactivación se administró Propranolol, lo que provocó una reducción de la respuesta fisiológica y del miedo auto-percibido hacia estímulos relacionados con los arácnidos; esta reducción prevaleció, por lo menos, durante un año solo en aquel grupo que había recibido la reactivación seguido del Propranolol y no así en los grupos que recibieron placebo o el fármaco en ausencia de reactivación. Más aún, el tratamiento perjudicó la reestabilización de la respuesta de miedo autonómica, pero el contenido declarativo de la misma quedó intacto (Soeter y Kindt, 2015).

Es importante recalcar los recientes trabajos de Chalkia et al. $(2019 ; 2020)$ que pusieron en duda los resultados obtenidos en los paradigmas antes mencionados; especialmente el estudio de Schiller (2010) y el llevado a cabo por Soeter y Kindt (2015). Chalkia replicó los procedimientos y falló en encontrar los mismos resultados exponiendo así la importancia de los criterios que el evaluador utiliza y los paradigmas creados para provocar la reactivación de la memoria aversiva. Estos resultados ponen en evidencia la necesidad de investigar en profundidad los 
diferentes factores que pueden influir en la reconsolidación, para luego desarrollar una terapia efectiva basada en este proceso.

Así, la reconsolidación representaría un proceso único que posibilita la modificación de la información almacenada, pudiendo ser utilizado como herramienta en la clínica en combinación con las terapias actuales. En el caso de memorias maladaptativas con componentes emocionales, implicaría la modificación de los componentes autonómicos -que llevan a una respuesta automática excesiva-, aunque conservando el acceso al contenido episódico.

Sin embargo, hay que tener en cuenta que cuando hablamos de memorias autobiográficas, factores limitantes del proceso de reconsolidación, como la edad de la memoria (por ejemplo, una memoria antigua podría ser una memoria de nuestra infancia) y la fuerza de la misma (por ejemplo, una memoria fuerte podría ser aquella que fue evocada repetidas veces) podrían dificultar la iniciación de este proceso (Fernández et al., 2016a).

\section{Reactivación de memorias durante el sueño}

Para desarrollar terapias combinadas que involucren la modificación de memorias almacenadas no puede dejarse de lado el estudio del sueño. Como hemos discutido previamente, el sueño cumple un rol fundamental en el reforzamiento e integración de nueva información, así como en el reprocesamiento del tono emocional de las memorias y como regulador de la homeostasis sináptica que permite nuevas rondas de codificación (Rasch y Born, 2013; Tononi y Cirelli, 2008; Walker y Van der Helm, 2009). Si bien durante el sueño se producen reactivaciones espontáneas de la memoria (Ji y Wilson, 2007), las mismas pueden inducirse a través de la presentación de claves ligadas a la experiencia. Así, las reactivaciones inducidas durante el sueño profundo mejoran la consolidación y reconsolidación de la memoria declarativa (Diekelmann et al., 2011; Klinzing et al., 2016; Moyano et al., 2019; Rudoy et al., 2009; Rasch et al., 2007). Uno de los estudios que revolucionó el campo del sueño y la memoria fue el realizado por Rasch et al. (2007). Los participantes debían aprender una tarea de localización espacial de objetos -lo que conocemos como "memotest"-, en presencia de un olor a rosas. Luego durmieron ocho horas mientras se les realizaba una polisomnografía y se les presentó el olor a rosas (recordatorio) o solución fisiológica (control) en diferentes fases de sueño. A la mañana siguiente, durante la evaluación, se observó que el grupo que fue entrenado con el olor a rosas y recibió el mismo olor durante el sueño de ondas lentas, mejoró significativamente su memoria comparado con el grupo control que recibió solución fisiológica. Este reforzamiento no se observó cuando el recordatorio fue presentado en sueño MOR, en la vigilia anterior al período de sueño, o si se presentaba el olor a rosas durante el sueño de ondas lentas pero el mismo no fue asociado al aprendizaje previo (Rasch et al., 2007). Este estudio fue uno de los primeros en demostrar cómo puede inducirse la modificación de una memoria declarativa mientras dormimos y en apoyar la idea de que las reactivaciones espontáneas de la memoria durante el sueño favorecen la consolidación de la información almacenada. 
Por otro lado, hay que tener en cuenta que la reactivación de la memoria declarativa durante la vigilia puede llevar a la labilización/reconsolidación de la memoria. Así, a una memoria que es labilizada durante la vigilia le puede llevar alrededor de seis horas restablecerse (Forcato et al., 2007). Sin embargo, si la reactivación es seguida por un período de sueño, -por ejemplo, una siesta de $90 \mathrm{~min}$. -, resulta suficiente para restablecer la memoria. Es decir, el sueño favorece la reconsolidación de la misma, acelerando el proceso y protegiéndola contra interferencias (Moyano et al., 2019).

La posibilidad de reactivar memorias durante el sueño para guiar su modificación hace pensar acerca de su utilidad en el diseño de herramientas para su aplicación en la clínica, tanto para perjudicar la memoria maladaptativa como para mejorar la incorporación de las intervenciones terapéuticas durante el sueño.

Así, Groch et al. (2017) indujeron reactivaciones durante el sueño a adolescentes con diagnóstico de Trastorno de Ansiedad (TA), como también a un grupo control de adolescentes sin dicho trastorno. Los participantes debían observar imágenes relativamente ambiguas pero comunes de la vida diaria y debían evocar de forma libre emociones o descripciones acerca de las mismas. A su vez, se les pidió que imaginaran estar viviendo la situación representada en la imagen y que la describieran con una palabra. Luego, se les permitió dormir ocho horas mientras se les realizaba una polisomnografía. La memoria fue reactivada en sueño NoMOR a través de la presentación de la mitad de las palabras elegidas por cada participante y fue finalmente evaluada al despertar, así como también una semana después. Para ello, se presentaron las imágenes y los sujetos debían evocar la palabra asignada y calificar afectivamente cada una de ellas con una escala que establecía grados entre muy desagradable y muy emocionante. Se observó entonces que la reactivación mejoraba la consolidación de la información reactivada para ambos grupos. Por otro lado, la reactivación no afectó significativamente los sentimientos evocados en la primera evaluación -realizada al despertar-, pero sí afectó de manera significativa la evocación de sentimientos desagradables o negativos luego de una semana. Más específicamente, la reactivación se asoció a una reducción del afecto asociado a la palabra, cuando ésta era inicialmente negativa en pacientes con TA. Esto no fue observado en los participantes del grupo control. A su vez, la reactivación en estos pacientes fue asociada a un aumento de la evaluación afectiva de las imágenes descriptas positivamente, pero no en los sujetos del grupo control. También se observó, solo en pacientes con TA, un aumento significativo en la potencia en las bandas de frecuencia correspondientes a las oscilaciones theta (4-8 Hz) durante la reactivación de las memorias negativas. La potencia en theta correlacionó positivamente con el desempeño de aquellas memorias reactivadas durante el sueño. Groch y colaboradores plantean que estas diferencias se deben a que las memorias reactivadas se recuerdan mejor si existe una red preexistente a la que puede asociarse. En el caso de los pacientes con TA, que suelen recordar con mayor facilidad pensamientos o recuerdos negativos autobiográficos, la integración a redes prexistentes de las imágenes que ellos mismos consideraban negativas o desagradables no sólo se vio favorecida, sino que ayudó a que decreciese la 
valoración afectiva asociada a la misma (Groch et al., 2017). Los autores plantean que los mecanismos que hacen que el sueño favorezca la consolidación de la memoria también disminuyen el tono emocional de la misma, al igual que plantean Walker y Van der Helm (2009) en relación al sueño MOR.

Anteriormente, hemos discutido que la terapia de exposición es una de las más utilizadas para el tratamiento de memorias maladaptativas como las fobias (Foa y Kozak, 1986). Cuando la terapia funciona, suele ser una intervención efectiva en fobias; sin embargo, como hemos visto, los síntomas de algunos pacientes aminoran durante un tiempo, para luego retornar (Kleim et al., 2014). Esta recuperación espontánea de la memoria original con el simple paso del tiempo, pone aún más en evidencia que en este tipo de tratamientos estaría involucrado el proceso de extinción. Así, si pensamos la terapia de exposición como un nuevo aprendizaje que necesita consolidarse e integrarse a la información previamente almacenada, el sueño podría tener un rol activo en este proceso y por lo tanto ser de utilidad en el diseño de una terapia. En la misma línea, Kleim et al. (2014) estudiaron el efecto del sueño posterior a una terapia de exposición virtual, en pacientes que sufrían de fobia a las arañas. Luego de la terapia de exposición los participantes fueron divididos en dos grupos. A un grupo se le hizo dormir una siesta de 90 minutos; el otro permaneció despierto durante el mismo período de tiempo. Luego fueron evaluados en materia de ansiedad subjetiva y cogniciones relacionadas por los arácnidos (pensamientos generados por las arañas), a través de una entrevista estructurada y cuestionarios diversos. La evaluación se repitió una semana después para comprobar que los efectos de la terapia de exposición continuarán haciendo efecto. Los investigadores encontraron que, para el grupo experimental que había dormido una siesta tras la terapia de exposición virtual, los síntomas fóbicos eran significativamente menores que los del grupo que permaneció despierto. Estos resultados indican que es recomendable que los pacientes ansiosos duerman después de la terapia, ya que podría ayudar a favorecer los efectos terapéuticos. Ahora bien, en este punto, como discutiremos más adelante, hay que tener precaución, dado que el sueño no solo puede reforzar e integrar la terapia, sino que también puede reforzar la memoria original perjudicial y desencadenar así una respuesta maladaptativa mayor.

Como hemos visto, el sueño influye activamente en los procesos de memoria y el mismo se caracteriza tanto por la actividad eléctrica oscilatoria como neuromodulatoria (Diekelmann y Born, 2010). En algunos trastornos, como en el Trastorno por Estrés Post Traumático, existe una desregulación del ciclo de sueño. Vanderheyden et al. (2014) intentaron dilucidar cómo opera el sueño en este trastorno. Plantearon que las estructuras más importantes, implicadas en el sueño y en el trastorno de estrés postraumático, son: 1) la amígdala, implicada en la codificación, el guardado y la recuperación de las memorias asociadas al miedo así como también en la activación del sistema nervioso autónomo, encargado de la ejecución de la reacción automática ante el miedo y responsable de respuestas tales como: la dilatación de pupilas, el aumento del ritmo cardíaco, la respiración, etc.; 2) El hipocampo y la corteza prefrontal, estructuras fundamentales en procesos que involucran a la memoria. 
Las tres estructuras mencionadas se encargan de la modulación contextual de las memorias traumáticas. A su vez, la actividad noradrenérgica (NA), que está ligada al procesamiento de memorias emocionales, se encuentra elevada durante el sueño MOR cuando normalmente su actividad en dicha fase de sueño es nula (Rasch y Born, 2013). Así, la mínima actividad Noradrenérgica durante el sueño MOR permitiría agregar, a una memoria episódica existente, nueva información adquirida durante el día en esquemas más estables de la corteza prefrontal. En los sujetos con TEPT, la actividad del locus cerúleo (principal fuente de NA del cerebro), que secreta constantemente noradrenalina durante el sueño MOR, favorece únicamente la potenciación a largo plazo de la memoria traumática original y, por lo tanto, la capacidad de incorporar nuevas memorias a una red ya formada se ve imposibilitada. Al mismo tiempo, la inhibición que normalmente ocurre desde la corteza prefrontal hacia la amígdala se ve perjudicada en el TEPT deteriorando el proceso mediante el cual se codifica el trauma. Por último, Vanderheyden et al. (2014) hacen además referencia a la hiperactivación de la amígdala en el TEPT, que actúa como feedback para sostener, a su vez, la actividad del locus cerúleo. En resumen, todo este circuito desregulado genera, por un lado, una hiperactivación ante claves que podrían asociarse al evento traumático en pacientes con TEPT y, por el otro, la imposibilidad de agregar nueva información a memorias ya existentes. Esto último, en términos clínicos, se traduce en una dificultad a la hora de modificar una memoria aversiva muy presente en el sujeto con TEPT (Vanderheyden et al., 2014).

Dado que el sueño favorece la consolidación de memorias tanto neutras como emotivas ¿será posible ir un paso antes e inhibir el desarrollo de estrés post-traumático a partir de la privación de sueño de personas que han sufrido un evento traumático? Kuriyama et al. (2010) investigaron el impacto de una noche de privación de sueño luego de un evento aversivo en la consolidación de esa memoria. Los participantes observaron videos tanto aversivos como neutros (filmaciones recogidas de taxis de la ciudad de Tokio en donde unas mostraban choques contra peatones o ciclistas y otras sólo recorridos cotidianos sin desenlaces fatales); luego, a uno de los grupos se los privó la primera noche de sueño, mientras que al otro grupo se le permitió dormir normalmente. Los resultados obtenidos demostraron que el grupo que fue privado de sueño redujo significativamente las respuestas fisiológicas de miedo que provocaron las imágenes aversivas presentadas como también la generalización de respuesta hacia los otros estímulos, mientras que el grupo de sueño generalizó las respuestas de miedo de una manera más rápida y no redujo las respuestas fisiológicas de miedo suscitadas por las imágenes (Kuriyama et al., 2010).

Un punto interesante a tener en cuenta es que los propios autores reconocen que el grupo privado de sueño tuvo una noche de sueño intermedia antes de la evaluación, lo que pudo haber provocado una recuperación en el sueño y una subsecuente consolidación de la memoria aversiva igual a la del grupo que sí durmió. Pero los resultados demostraron lo contrario, sugiriendo que las memorias emotivas dependientes de la amígdala son más susceptibles a la privación de sueño que las memorias declarativas dependientes del hipocampo (Kuriyama et al., 2010). Si bien 
este estudio habla de un único evento aversivo y no menciona nada sobre el cuadro clínico de TEPT -que se encuentra presente durante mucho tiempo, generando trastornos del ciclo de sueño como el insomnio-, no deja por ello de exponer el rol central del sueño en la consolidación de memorias aversivas.

\section{Discusión}

Esta revisión fue creada con el objetivo de exponer, de forma abreviada, cuál es el estado del arte de la consolidación y reconsolidación de memorias maladaptativas y su relación con el sueño. A partir de lo expuesto, podemos delinear algunos parámetros para pensar estrategias dentro del ámbito clínico en dónde se tengan en cuenta los avances en materia de reactivación de memoria.

Consideramos necesario que el diseño de nuevas terapias combine las utilizadas actualmente en el campo de la psicología con los crecientes hallazgos de las neurociencias en el campo de la modificación de memorias. Para eso hay que tener en cuenta: 1) las características de la memoria original como su antigüedad, su fuerza y su tipo; 2) el tipo de reactivación inducida, así como el momento de la reactivación con respecto al período de sueño; 3) el tratamiento posterior a la reactivación.

A partir de los hallazgos anteriormente mencionados, una memoria reactivada puede sufrir tres destinos: 1) puede reforzarse, es decir aumentar la precisión de la memoria y/o la persistencia de la misma; 2) puede perjudicarse su reestabilización; 3) puede incorporarse nueva información dentro de la misma red de memoria, ya sea por la introducción de nuevo material, o modificando, por ejemplo, el significado del estímulo aprendido (Forcato et al., 2007, 2010, 2011, 2013; Schiller et al., 2010). Dentro de este último caso, podemos pensar las reactivaciones que son seguidas por un aprendizaje de extinción (Schiller et al., 2010). Este detalle no es menor, ya que a la hora de diseñar un plan de tratamiento el profesional pone como objetivo producir un cambio de aquello que aqueja al sujeto. Hay que prestar máxima atención al hecho de no reforzar una memoria maladaptativa, ya que, con solo una reactivación, la misma puede aumentar su persistencia (Forcato et al., 2013). Es por eso que resulta de vital importancia definir el tratamiento posterior a la reactivación. Por ejemplo, en el caso de una memoria traumática, como la memoria de un choque automovilístico. Al momento de ser tratada, se debe tener en mente qué intervenciones realizar para evitar una mera reactivación de la misma, ya que eso llevaría al sujeto a formar trazas mnésicas aún más fuertes que lo lleven a un agravamiento del cuadro clínico. El objetivo de la intervención, en el caso de una memoria producida en un choque automovilístico, tendría que estar orientado entonces a la actualización y modificación de los aspectos emocionales negativos suscitados por la misma.

Esta consideración también debería tenerse en cuenta en el caso de los tratamientos para fobias específicas; por ejemplo, en casos donde podríamos estar enfrentándonos a la creación de nuevas memorias de extinción que compiten con la memoria original 
a la hora de expresarse. Esta advertencia no es menor, ya que no tener en cuenta este aspecto puede llevar a que la memoria original aversiva tome nuevamente control de la conducta del sujeto. En cambio, si se plantean protocolos de extinción que puedan aplicarse luego de reactivar y labilizar la memoria fóbica, se puede pensar en un uso más estratégico del contexto terapéutico. Esto permitiría modificar los aspectos autonómicos que surgen a partir de la presentación del estímulo aversivo, pero conservando aspectos episódicos de la memoria.

Dentro del marco de las recomendaciones, es importante tener en cuenta las claves que habrán de utilizarse en el proceso de la reactivación de la memoria. Tal como vimos en el estudio llevado a cabo por Rasch et al. (2007), una clave olfativa puede funcionar como modo de reactivación de la memoria, fortaleciéndola durante el sueño. De esta manera, se puede pensar un tratamiento en el cual se reactive la memoria aversiva para luego trasladar al sujeto a otro contexto (dentro de la ventana temporal de reconsolidación de esa memoria); en este nuevo contexto debe encontrarse una clave (por ejemplo, una clave olfativa, como un olor a rosas) y allí realizar una intervención que disminuya la ansiedad provocada por la reactivación de la memoria aversiva. Así, el sujeto asocia esta nueva memoria con un estímulo olfativo que podrá ser utilizado como recordatorio durante el posterior sueño de ondas lentas, fortaleciendo así su consolidación e integración.

Ahora bien, ya vimos el rol principal del sueño en la formación de memorias y también la importancia que tiene para reducir su tono emocional (Rasch y Born, 2013; Walker y Van der Helm, 2009). Teniendo en cuenta el trabajo de Kuriyama et al. (2010), en donde la privación de sueño posterior al entrenamiento de videos aversivos reducía la respuesta fisiológica de miedo, podría pensarse también en intervenciones de privación de sueño, con fármacos que eviten el rebote de sueño, para así disminuir la generación de memorias traumáticas.

Esta revisión tuvo como objetivo reforzar la importancia de entender cómo el aporte de las neurociencias se vuelve imprescindible para el desarrollo de la psicología y viceversa. No es posible sostener el dualismo cartesiano en donde la "mente" o "alma" se encuentra separada del "cuerpo" o del "cerebro". Por el contrario, el ser humano deber ser contemplado de forma holística y abarcado como un objeto de estudio único, en donde las distintas disciplinas realicen sus aportes desde una perspectiva interdisciplinaria y, por lo tanto, más enriquecedora. Temáticas tales como los tratamientos terapéuticos podrían ser pensados de una forma mucho más completa, pudiendo ofrecer, de este modo, un tratamiento más efectivo. 


\section{Referencias}

Alberini, C. M. y Ledoux, J. E. (2013). Memory reconsolidation. Current Biology, 23, R746-R750. HTTPS://DOI.ORG/10.1016/J.CUB.2013.06.046

American Psychiatric Association (2013). DSM5. Diagnostic and statistical manual of mental disorders (5ta edición). APA

American Psychological Association, division 12. (8 de junio de 2020). Exposure therapies for specific phobias status: strong research support. HTTPs:// W W W. DIV12.ORG/TREATMENT/EXPOSURETHERAPIES-FOR-SPECIFIC-PHOBIAS/

Anderson, M. C. y Hanslmayr, S. (2014). Neural mechanisms of motivated forgetting. Trends in Cognitive Sciences, 18(6), 279-292. HTTPs:// DX.DOI.ORG/10.1016\%2FJ.TICS.2014.03.002

Anderson, M. C., Bjork, R. A. y Bjork, E. L. (1994) Remembering can cause forgetting: retrieval dynamics in long-term memory. Journal of Experimental Psychology: Learning, Memory and Cognition, 20(5), 1063-1087.

Beck, A. T. (1985). Theoretical perspectives on clinical anxiety. LEA

Berger, R. J. y Phillips, N. H. (1995). Energy conservation and sleep. Behavioral and Brain Sciences, 69(1-2), 65-73. HTTPs://DOI. ORG./10.1016/0166-4328(95)00002-B

Borbély, A. A., Daan, S., Wirz-Justice, A. y Deboer, T. (2016). The two-process model of sleep regulation: a reappraisal. Journal of Sleep Research, 25(2), 131-143. HTTPS://DOI.ORG/10.1111/JSR.12371

Born, J. y Fehm, H.L. (1998). Hypothalamuspituitary-adrenal activity during human sleep: a coordinating role for the limbic hippocampal system. Experimental and Clinical Endocrinology and Diabetes, 106,153-163. HTTP://DX.DOI. ORG/10.1055/s-0029-1211969

Born, J. y Wilhelm, I. (2012). System consolidation of memory during sleep. Psychological Research, 76(2), 192-203. HTTPs://DOI.ORG/10.1007/soo426011-0335-6

Bouton M. E. (2002). Context, ambiguity, and unlearning: sources of relapse after behavioral extinction. Biological Psychiatry, 52(10), 976-986. HTTPS://DOI.ORG/10.1016/s0006-3223(02)01546-9

Braun, A. R., Balkin, T. J., Wesenten, N. J., Carson, R. E., Varga, M., Baldwin, P., Selbie, S., Belenky, G. y Herscovitch, P. (1997). Regional cerebral blood flow throughout the sleep-wake cycle. An H2(15) O PET study. Brain, 120(7), 1173-1197. HTTPs:// DOI.ORG/10.1093/BRAIN/120.7.1173
Brunet, A., Orr, S. P., Tremblay, J., Robertson, K., Nader, K. y Pitman, R. K. (2008). Effect of postretrieval propranolol on psychophysiologic responding during subsequent script-driven traumatic imagery in post-traumatic stress disorder. Journal of Psychiatric Research, 42(6), 503-506. HTTPS://DOI.ORG/10.1016/J. JPSYCHIRES.2007.05.006

Buchsbaum, M. S., Gillin, J. C., Wu, J., Hazlett, E., Sicotte, N., Dupont, R. M. y Bunney, W. E., Jr (1989). Regional cerebral glucose metabolic rate in human sleep assessed by positron emission tomography. Life Sciences, 45(15), 1349-1356. HTTPS://DOI.ORG/10.1016/0024-3205(89)90021-0

Buzsáki, G. (1989). Two-stage model of memory trace formation: a role for "noisy" brain states. Neuroscience, 31(3), 551-570.

Chalkia A, Weermeijer J, Van Oudenhove L and Beckers T (2019) Acute but not permanent effects of propranolol on fear memory expression in humans. Front. Hum. Neurosci., 13, 51. HтTPs:// DX.DOI.ORG/10.3389/FNHUM.2019.00051

Chalkia, A., Van Oudenhove, L. y Beckers, T. (2020). Preventing the return of fear in humans using reconsolidation update mechanisms: A verification report of Schiller et al.(2010). Cortex, 129, 510-525. HTTPs://DOI.ORG/10.1016/J. CORTEX.2020.03.031

Cirelli C, Tononi G (2008) Is sleep essential? PLoS Biol, 6(8), e216. HTTPS://DOI.ORG/10.1371/ JOURNAL.PBIO.0060216

Clark, D.D y Beck, A. T. (2012). Terapia cognitiva para trastornos de ansiedad. Descleé de Brouwer.

Diekelmann, S. y Born, J. (2010). The memory function of sleep. Nature Reviews Neuroscience, 11(2), 114-126. HTTPS://DOI.ORG/10.1038/NRN2762

Diekelmann, S., Büchel, C., Born, J. y Rasch, B. (2011). Labile or stable: opposing consequences for memory when reactivated during waking and sleep. Nature Neuroscience, 14(3), 381-386. HTTPS://DOI.ORG/10.1038/NN.2744

Dudai, Y. (2004). Memory from A to Z: Keywords, concepts, and beyond. Oxford University Press.

Dudai, Y. y Morris, R.G.M. (2000). To consolidate or not to consolidate: What are the questions? In J. J. Bluhuis (Ed.), Brain, perception, memory. Advances in cognitive sciences (pp. 149-162). Oxford University Press. 
Duss, S. B., Reber, T. P., Hänggi, J., Schwab, S., Wiest, R., Müri, R. M., ... y Henke, K. (2014). Unconscious relational encoding depends on hippocampus. Brain, 137(12), 3355-3370.

Ebbinghaus, D. (1885). Uber das Gedachtnis. Dunker y Humbolt.

Ekman, P. y Friesen, W. V. (2003). Unmasking the face: A guide to recognizing emotions from facial clues. ISHK. Malor Books.

Fernández, R. S., Bavassi, L., Forcato, C. y Pedreira, M. E. (2016a). The dynamic nature of the reconsolidation process and its boundary conditions: Evidence based on human tests. Neurobiology of Learning and Memory, 130, 202212. HTTPS://DOI.ORG/10.1016/J.NLM.2016.03.001

Fernández, R. S., Bavassi, L., Kaczer, L., Forcato, C y Pedreira, M. E. (2016b). Interference conditions of the reconsolidation process in humans: The role of valence and different memory systems. Frontiers in Human Neuroscience, 10, 641. HTTPS://DOI.ORG/10.3389/FNHUM.2016.00641

Foa, E. B. y Kozak, M. J. (1986). Emotional processing of fear: exposure to corrective information. Psychological Bulletin, 99(1), 20.

Forcato, C., Argibay, P. F., Pedreira, M. E. y Maldonado, H. (2009). Human reconsolidation does not always occur when a memory is retrieved: the relevance of the reminder structure. Neurobiology of Learning and Memory, 91(1), 5057. HTTPS://DOI.ORG/10.1016/J.NLM.2008.09.011

Forcato, C., Burgos, V. L., Argibay, P. F., Molina, V. A., Pedreira, M. E. y Maldonado, H. (2007). Reconsolidation of declarative memory in humans. Learning and Memory, 14(4), 295-303. HTTPS://DOI.ORG/10.1101/LM.486107

Forcato, C., Fernandez, R. S. y Pedreira, M. E. (2013). The role and dynamic of strengthening in the reconsolidation process in a human declarative memory: what decides the fate of recent and older memories? PLoS One, 8(4), e61688. HTTPs://DOI. ORG/10.1371/JOURNAL.PONE.0061688

Forcato, C., Rodríguez, M. L. y Pedreira, M. E. (2011) Repeated labilization-reconsolidation processes strengthen declarative memory in humans. PLoS One, 6(8), e23305. HTTPS://DOI.ORG/10.1371/ JOURNAL.PONE.0023305

Forcato, C., Fernández, R. S. y Pedreira, M. E. (2014) Strengthening a consolidated memory: The key role of the reconsolidation process. Journal of Physiology - Paris, 108, 323-333. HTTPs://DOI. ORG/10.1016/J.JPHYSPARIS.2014.09.001.
Forcato, C., Rodríguez, M. L., Pedreira, M. E. y Maldonado, H. (2010). Reconsolidation in humans opens up declarative memory to the entrance of new information. Neurobiology of Learning and Memory, 93(1), 77-84. HTTPs://DOI. ORG/10.1016/J.NLM.2009.08.006

Groch, S., Preiss, A., McMakin, D. L., Rasch, B., Walitza, S., Huber, R. y Wilhelm, I. (2017). Targeted reactivation during sleep differentially affects negative memories in socially anxious and healthy children and adolescents. Journal of Neuroscience, 37(9), 2425-2434. HTTPS://DOI. ORG/10.1523/JNEUROSCI.1912-16.2017

Henke, K. (2010). A model for memory systems based on processing modes rather than consciousness. Nature Reviews Neuroscience, 11(7), 523-532.

Hobson, J. A., Pace-Schott, E. F. y Stickgold, R. (2000). Dreaming and the brain: toward a cognitive neuroscience of conscious states. Behavioral and Brain Sciences, 23, 793-842. HTTPs://DOI. ORG./10.1017/S0140525X00003976

Hupbach, A., Gómez, R., Hardt, O. y Nadel, L. (2007). Reconsolidation of episodic memories: a subtle reminder triggers integration of new information. Learning and Memory, 14(1-2), 4753. HTTPS://DX.DOI.ORG/10.1101\%2FLM.365707

Jacobacci, F., Armony, J. L., Yeffal, A., Lerner, G., Amaro, E., Jovicich, J., ... y Della-Maggiore, V. (2020). Rapid hippocampal plasticity supports motor sequence learning. Proceedings of the National Academy of Sciences, 117(38), 2389823903.

Ji, D., Wilson, M. Coordinated memory replay in the visual cortex and hippocampus during sleep. Nat Neurosci, 10, 100-107 (2007). HTTPs://DOI. ORG/10.1038/NN1825

Kandel, E. R., Jessell, T. M., Schwartz J. H. (2000). Mecanismos celulares del aprendizaje y la memoria. Neurociencia y conducta. Prentice Hall.

Kleim, B., Wilhelm, F. H., Temp, L., Margraf, J., Wiederhold, B. K. y Rasch, B. (2014). Sleep enhances exposure therapy. Psychological Medicine, 44(7), 1511-1519. HTTPS://DOI. ORG/10.1017/so033291713001748

Klinzing, J. G., Rasch, B., Born, J. y Diekelmann, S. (2016). Sleep's role in the reconsolidation of declarative memories. Neurobiology of Learning and Memory, 136, 166-173. HTTPS://DOI. ORG/10.1016/J.NLM.2016.10.004

Knutson, K. L., Spiegel, K., Penev, P. y Van Cauter, E. (2007). The metabolic consequences of sleep deprivation. Sleep Medicine Reviews, 11(3), 163-178. HTTPS://DX.DOI.ORG/10.1016/J. SMRV.2007.01.002 
Kuriyama, K., Soshi, T. y Kim, Y. (2010). Sleep deprivation facilitates extinction of implicit fear generalization and physiological response to fear. Biological Psychiatry, 68(11), 991-998. HтTPs:// DOI.ORG/10.1016/J.BIOPSYCH.2010.08.015

Lane R. D., Ryan L., Nadel L. y Greenberg L. (2015) Memory reconsolidation, emotional arousal, and the process of change in psychotherapy: new insights from brain science. Behavioral and Brain Sciences, 38, el. HTTPS://DOI.ORG/10.1017/ s0140525X14000041

Lange, T., Dimitrov, S. y Born, J. (2010). Effects of sleep and circadian rhythm on the human immune system. Annals of the New York Academy of Sciences, 1193, 48-59. HTTPS://DX.DOI. ORG/10.1111/J.1749-6632.2009.05300.X

Marr, D. (1971). Simple memory: A theory for archicortex. Philos Trans R Soc Lond B Biol Sci. 262(841), 23- 81 .

McCarley, R.W. (2007). Neurobiology of REM and NREM sleep. Sleep Medicine, 8, 302-30. HTTPs:// DOI.ORG/10.1016/J.SLEEP.2007.03.005

McGaugh, J.L. (2004). The amygdala modulates the consolidation of memories of emotionally arousing experiences. Annual Review of Neuroscience, 27, 1-28. HTTPS://DOI.ORG/10.1146/ ANNUREV.NEURO.27.070203.144157

Moyano, M.D., Diekelmann, S., Pedreira, M.E., Born, J. y Forcato, C. (2019). Sleep accelerates re-stabilization of human declarative memories. Neurobiology of Learning and Memory, 162, 1-8. HTTPS://DOI.ORG/10.1016/J.NLM.2019.04.012

Myers, K.M. y Davis, M. (2002) Behavioral and neura analysis of extinction. Neuron, 36, 567-584. HTTPS://DOI.ORG/10.1016/s0896-6273(02)01064-4

Nader, K., Hardt, O., Einarsson, E. Ö. y Finnie, P. S. B. (2013). Chapter two - The Dynamic Nature of Memory. En C. M. Alberini (Ed.), Memory reconsolidation (pp. 15-41). Academic Press.

Nader, K., Schafe, G. E. y Le Doux, J. E. (2000) Fear memories require protein synthesis in the amygdala for reconsolidation after retrieval. Nature, 406(6797), 722-726. HTTPS://DOI. ORG/10.1038/35021052

Oswald I. (1980). Sleep as restorative process: human clues. Progress in Brain Research, 53, 279-288. HTTPS://DOI.ORG/10.1016/soo79-6123(08)60069-2

Pedreira, M. E., Pérez-Cuesta, L. M. y Maldonado, H. (2004). Mismatch between what is expected and what actually occurs triggers memory reconsolidation or extinction. Learning $y$ Memory, 11(5), 579-585. HTTPS://DX.DOI. ORG/10.1101\%2FLM.76904
Rasch, B. y Born, J. (2013). About sleep's role in memory. Physiological Reviews, 93(2), 681-766. HTTPS://DOI.ORG/10.1152/PHYSREV.00032.2012

Rasch, B., Büchel, C., Gais, S. y Born, J. (2007). Odor cues during slow-wave sleep prompt declarative memory consolidation. Science, 315(5817), 14261429. HTtPs://DOI.ORG/10.1126/SCIENCE.1138581

Reber, T. P., Luechinger, R., Boesiger, P. y Henke, K. (2014). Detecting analogies unconsciously. Frontiers in Behavioral Neuroscience, 8, 9.

Reber, T. P. y Henke, K. (2011). Rapid formation and flexible expression of memories of subliminal word pairs. Frontiers in Psychology, 2, 343.

Rechtschaffen, A. y Bergmann, B. M. (1995). Sleep deprivation in the rat by the disk-overwater method. Behavioral and Brain Sciences, 69(1-2), 55-63. HTTPs://DOI.orG/10.1016/01664328(95)00020-T

Rechtschaffen, A. y Kales, A. (1968). A manual of standardized terminology, technique and scoring system for sleep stages of human sleep. UCLA.

Rudoy, J. D., Voss, J. L., Westerberg, C. E. y Paller, K. A. (2009). Strengthening individual memories by reactivating them during sleep. Science, 326(5956), 1079. HTTPS://DOI.ORG/10.1126/ SCIENCE. 1179013

Schiller, D., Monfils, M-H., Raio, C. M., Johnson, D.C., Ledoux, J.E. y Phelps, E.A. (2010). Preventing the return of fear in humans using reconsolidation update mechanisms. Nature, 463, 49-53. HTTPS://DOI.ORG/10.1038/NATUREO8637

Schwabe, L., Nader, K., Wolf, O.T., Beaudry, T. y Pruessner, J. C. (2012). Neural signature of reconsolidation impairments by propranolol in humans. Biological Psychiatry, 71(4), 380冈386. HTTPS://DOI.ORG/10.1016/J.BIOPSYCH.2011.10.028

Schwabe, L. y Wolf, O.T. (2009). New episodic learning interferes with the reconsolidation of autobiographical memories. Plos ONE, 4(10), e7519. HTTPS://DOI.ORG/10.1371/JOURNAL. PONE.0007519.

Sevenster, D., Beckers, T. y Kindt, M. (2013). Prediction error governs pharmacologically induced amnesia for learned fear. Science, 339(6121), 830-833. HTTPS://DOI.ORG/10.1126/ SCIENCE. 1231357

Sinclair AH, Barense MD. (2019). Prediction error and memory reactivation: How incomplete reminders drive reconsolidation. Trends Neurosci., 42(10), 727-739. 
Soeter, M. y Kindt, M. (2015). An abrupt transformation of phobic behavior after a postretrieval amnesic agent. Biological Psychiatry, 78, 880-886. HTTPS://DOI.ORG/10.1016/J. BIOPSYCH.2015.04.006

Squire, L. R. y Dede, A. J. (2015). Conscious and unconscious memory systems. Cold Spring Harbor Perspectives in Biology, 7(3), a021667.

Steriade, M., Timofeev, I. y Grenier, F (2001). Natural waking and sleep states: a view from inside neocortical neurons. Journal of Neurophysiology, 85(5), 1969-1985. HTTPS://DOI.ORG./10.1152/ JN.2001.85.5.1969

Tononi G. y Cirelli C. (2006). Sleep function and synaptic homeostasis. Sleep Medicine Reviews, 10, 49-62. HTTPS://DOI.ORG/10.1016/J. SMRV.2005.05.002

Tononi, G. y Cirelli, C. (2014). Sleep and the price of plasticity: from synaptic and cellular homeostasis to memory consolidation and integration. Neuron, 81, 12-34. HTTPS://DOI.ORG/10.1016/J. NEURON.2013.12.025

Tulving, E. (1972). Episodic and semantic memory. Organization of Memory, 1, 381-403.

Van Cauter, E., Spiegel, K., Tasali, E. y Leproult, R. (2008). Metabolic consequences of sleep and sleep loss. Sleep Medicine, 9, S23-28. HTTPs://DOI. ORG/10.1016/s1389-9457(08)70013-3

Van der Helm E. y Walker M. P. (2010). Sleep and emotional memory processing. Sleep Medicine Clinics, 6, 31-43. HTTPS://DOI.ORG/10.1016/J. JSMC.2010.12.010
Vanderheyden, W. M., Poe, G. R. y Liberzon, I. (2014). Trauma exposure and sleep: using a rodent model to understand sleep function in PTSD. Experimental Brain Research, 232(5), 1575-1584. HTTPS://DOI.ORG/10.1007/soo221-014-3890-4

Varga A. W., Wohlleber M. E., Giménez S., Romero S., Alonso J. F., Ducca E. L., et al. (2016). Reduced slow-wave sleep is associated with high cerebrospinal fluid abeta42 levels in cognitively normal elderly. Sleep, 39, 2041-2048. нттPs:// DOI.ORG/10.5665/SLEEP.6240

Velázquez-Moctezuma, J., Shalauta, M., Gillin, J.C. y Shiromani, P. J. (1991). Cholinergic antagonists and REM sleep generation. Brain Research, 543, 175-179. HTTPS://DOI.ORG/10.1016/00068993(91)91064-8

Vorster, A. P. y Born, J. (2015). Sleep and memory in mammals, birds and invertebrates. Neuroscience y Biobehavioral Reviews, 50, 103-119. нттрs:// DOI.ORG/10.1016/J.NEUBIOREV.2014.09.020

Walker, M. P. y van der Helm, E. (2009). Overnight therapy? The role of sleep in emotional brain processing. Psychological Bulletin, 135(5), 731-48. HTTPS://DOI.ORG/10.1037/A0016570

Webb, W. B. (1988). An objective behavioral model of sleep. Sleep: Journal of Sleep Research $y$ Sleep Medicine, 11(5), 488-496. HTTPs://DOI. ORG/10.1093/SLEEP/11.5.488

Wuethrich, S., Hannula, D. E., Mast, F. W. y Henke, K. (2018). Subliminal encoding and flexible retrieval of objects in scenes. Hippocampus, 28(9), 633-643. 